\title{
Disparities in Hypertension Control Across and Within Three Health Systems Participating in a Data-Sharing Collaborative
}

\author{
Kevin Selby, MD, MAS, Martha Michel, PhD, Ginny Gildengorin, PhD, \\ Leah Karliner, MD, Rajiv Pramanik, MD, Valy Fontil, MD, MAS, \\ and Michael B. Potter, MD
}

Introduction: We aimed to standardize data collection from 3 health systems (HS1, HS2, HS3) participating in the San Francisco Bay Collaborative Research Network, and compare rates and predictors of uncontrolled blood pressure among hypertensive adults to identify opportunities for regional collaboration in quality improvement.

Methods: Retrospective cohort study using deidentified electronic health record data from all primary care patients with at least 1 visit in a 2-year period, using standard data definitions in a common data repository. Primary outcome was uncontrolled blood pressure at the most recent primary care visit.

Results: Of 169,793 adults aged 18 to 85 years, $53,133(31.3 \%)$ had a diagnosis of hypertension. Of these, 18,751 (35\%) had uncontrolled blood pressure at their last visit, with the proportion varying by system (29\%, HS1; 31\%, HS2; and 44\%, HS3) and by clinical site within each system. In multivariate analyses, differences between health systems persisted, with HS2 and HS3 patients having a 1.15 times (95\% CI, 1.11 to 1.19$)$ and 1.46 times (95\% CI, 1.42 to 1.50) greater relative risk of uncontrolled blood pressure compared with HS1. Across health systems, hypertensive patients were more likely to have uncontrolled blood pressure if they were uninsured, African Americans, current smokers, obese, or had fewer than 2 primary care visits during the 2 -year measurement period.

Conclusions: After controlling for standard individual predictors of hypertension control, significant and substantial differences in hypertension control persisted between health systems, possibly due to local quality improvement programs among other factors. There may be opportunities to share best practices and address common disparities across health systems. (J Am Board Fam Med 2018;31:897-904.)

Keywords: Blood Pressure, Hypertension, Multivariate Analysis, Primary Health Care, Retrospective Studies Quality Improvement

Health systems in the United States frequently use electronic patient registries to identify and track individual patients due for preventive care and co-

This article was externally peer reviewed.

Submitted 5 June 2018; revised 29 August 2018; accepted 30 August 2018.

From the Kaiser Permanente Division of Research, Oakland, CA (KS); Department of Ambulatory Care and Community Medicine, University of Lausanne, Switzerland (KS); Department of Family and Community Medicine, University of California-San Francisco, San Francisco (MM, GG, RP, MBP); Division of General Internal Medicine, Multiethnic Health Equity Research Center, University of California-San Francisco, San Francisco (LK, VF); UCSF Center for Vulnerable Populations at Zuckerberg San Francisco General Hospital, San Francisco (VF).

Funding: This research was supported by the National Center for Advancing Translational Sciences, National ordinate outreach, but less often to identify and address population-level disparities in outcomes. ${ }^{1}$ Similarly, health systems focusing on quality improvement for their own patients may overlook health disparities within their larger communities or fail to address these disparities in partnership

Institutes of Health (UL1 TR001872). Dr. Selby was supported by the Swiss Cancer Research Foundation (BIL KFS3720-08-2015).

Conflict of interest: none declared.

Prior presentations: North American Primary Care Research Group Annual Meeting, Montreal, Quebec, November 17-21, 2017.

Corresponding author: Michael B. Potter, MD, Department of Family and Community Medicine, University of California San Francisco School of Medicine, San Francisco, CA 94143-0900 (E-mail: Michael.potter@ucsf.edu). 
with other local stakeholders and health care providers. In 2014 the San Francisco Bay Collaborative Research Network, a primary care practice-based research network including academic, public, and private health care systems in northern California, convened health care leaders to explore better use of patient data. Stakeholders identified hypertension as a common and often undertreated condition. The Hypertension Data Collaborative was formed and supported with a small grant from the UCSF Clinical and Translational Science Institute.

Hypertension is a leading cause of preventable disease and death in the United States. ${ }^{2}$ In 2016, $29 \%$ of American adults had hypertension, and only $48 \%$ of hypertensive adults had blood pressure lower than 140/90 mm Hg. ${ }^{3}$ Hypertension prevalence increases with age and is higher among African Americans, whereas blood pressure control is better for non-Latino whites than for African Americans, Asian Americans, or Latinos. ${ }^{3}$ Those without insurance ${ }^{4}$ and without a regular primary care doctor ${ }^{5}$ are more likely to have uncontrolled blood pressure. Though some integrated health systems have improved hypertension diagnosis and management for their own patients, ${ }^{6}$ hypertension prevalence has remained constant since 2000, and blood pressure control has not substantially improved in the last decade. ${ }^{3}$

The 2014 Evidence-Based Guideline for the Management of High Blood Pressure in Adults introduced less stringent blood pressure targets $(<150 / 90 \mathrm{~mm} \mathrm{Hg})$ for adults aged 60 years and older without related comorbidities, ${ }^{7}$ while retaining uniform blood pressure targets for all others $(<140 / 90 \mathrm{~mm} \mathrm{Hg})$. In January 2017, the American Academy of Family Physicians and American College of Physicians issued their own evidence-based clinical guidelines and endorsed the 2014 guideline. ${ }^{8}$ Newer guidelines from the American College of Cardiology (ACC) and American Heart Association (AHA) ${ }^{9}$ have been met with skepticism, ${ }^{10}$ and American Academy of Family Physicians has officially decided against endorsing them. ${ }^{11}$ The goal of the UCSF Clinical and Translational Science Institute Hypertension Data Collaborative was, therefore, to use electronic health record (EHR) data to assess blood pressure control among patients diagnosed with hypertension across participating health systems, using the guidelines endorsed by primary care organizations in the United States. We examined similarities and differences across and within health systems as a first step toward regional collaboration to address hypertension disparities.

\section{Methods \\ Study Design and Setting}

This study was a retrospective, observational cohort analysis using 2 years of EHR data from affiliated health care systems. Seven health systems were invited to participate, 3 of which declined immediately due to competing priorities and insufficient internal resources to extract EHR data. Four health systems signed data-sharing agreements. Representatives from the 4 health systems met monthly over a 6-month period to create a shared data dictionary. One of these 4 health systems, a community clinic consortium, dropped out because of insufficient trained personnel and challenges getting independent affiliated clinics to agree to share their data within the timeline required by the project. The remaining 3 systems submitted deidentified data to a shared data repository. In meetings including clinicians familiar with the EHR and data definitions of each system, we created common definitions for all desired variables and then matched with available data from each EHR. A series of 8 meetings lasting 1 hour each was needed. The 3 health systems included 1 academic and 2 countyrun primary health care systems with a total of 31 primary care clinics. Two were using the Epic EHR, and 1 the eClinicalWorks EHR. The study was approved by the institutional review board with a waiver for informed consent. The 3 health systems are referred to as HS1, HS2, and HS3.

\section{Inclusion Criteria}

We included all patients aged 18 to 85 years at the beginning of the study period with a hypertension diagnosis and at least 1 primary care provider care visit between October 15, 2013 and October 14, 2015 at HS2 and HS3, or between May 1, 2014 and April 30, 2016 at HS1. The 2-year time period for HS1 was different from that for HS2 and HS3 due to local constraints and inability to pull data retrospectively to match the dates from HS2 and HS3. All information was collected using a 1-time extraction of EHR data. Hypertension diagnosis in each was defined using Ninth International Classification of Disease (ICD-9) visit diagnosis codes 401 to 405 for billing during the study time period or a 
problem list diagnosis indicating "hypertension." Exclusion criteria were pregnancy-related hypertension and end-stage renal disease.

\section{Variables}

The primary outcome was uncontrolled blood pressure at a patient's most recent primary care visit, defined as a blood pressure reading above the target set by 2014 guidelines. ${ }^{8}$ We stratified into 3 age groups, ages 18 to 39 years, 40 to 59 years, and 60 years and older, to match the 2014 guidelines and differentiate between younger and middleaged hypertensive patients. All patients aged 18 to 59 years and those $\geq 60$ to 85 years with ICD-9 visit diagnosis codes or problem-list diagnoses for guideline-relevant comorbidities (chronic kidney disease [CKD], diabetes mellitus [DM], and/or cardiovascular disease [CVD]) were defined as having uncontrolled hypertension if their diastolic pressure was $\geq 90 \mathrm{~mm} \mathrm{Hg}$ or systolic pressure $\geq 140$ mm Hg. ${ }^{8,9}$ CVD was defined as having an associated ICD-9 code for history of coronary artery disease, stroke, or transient ischemic attack. Although CVD was not included in the 2014 report as a guideline-relevant comorbidity, we chose to include this comorbidity because of the recommendation of the American College of Physicians and the American Academy of Family Physicians. ${ }^{8} \mathrm{~Pa}-$ tients aged 60 to 85 years without CKD, DM, or CVD were considered to have uncontrolled blood pressure if their diastolic pressure was $\geq 90 \mathrm{~mm} \mathrm{Hg}$ or systolic pressure $\geq 150 \mathrm{~mm} \mathrm{Hg}$.

We also collected age at first visit during the study period, sex, race and Latino ethnicity, body mass index (BMI; calculated from height and weight data), smoking status, preferred language, insurance status, and number of primary care visits during the 2-year study period. We combined race and Latino ethnicity, such that those recorded as both Latino ethnicity and either white or of unknown race were classified as Latino, while those recorded as both Latino ethnicity and either African American or Asian/Pacific Islander were classified as African-American or Asian/Pacific Islander.

\section{Analysis}

We report patient characteristics stratified by controlled and uncontrolled blood pressure using proportions. For the primary model, a generalized linear model with log link was used to identify risk factors for uncontrolled blood pressure after adjusting for confounders listed below. Generalized linear models estimate risk ratios instead of odds ratios, avoiding the misinterpretation of odds ratios and overestimation of risk for frequent outcomes. ${ }^{12}$ In a separate model, multivariate logistic regression was used to compare the probability of patients having uncontrolled blood pressure at each individual clinic in the 3 health systems after adjusting for confounders. Variables included in the 2 models were sex (male or female, as the 3 health systems did not record other gender categories); a nominal variable of age ( $<60$ or $\geq 60$ years); and the presence of 1 or more relevant comorbidities (CKD, $\mathrm{DM}$, or CVD); a combined variable for race and Latino ethnicity; insurance status (with uninsured and unknown combined, as 1 health system left health insurance unfilled for uninsured patients); smoking status at the most recent appointment (current or former/never); preferred language (English, Spanish, Chinese, or Other); obesity status (BMI $\geq 30$ or $<30 \mathrm{~kg} / \mathrm{m}^{2}$ ); number of primary care visits over the 2-year study period (1 to 2, 3 to 4 , or $\geq 5$ total visits); and health system where primary care was delivered (HS1, HS2, or HS3). Due to the relatively small number, we excluded patients with missing values $(\mathrm{N}=1726$ of 53,133$)$. We performed 4 sensitivity analyses. First, we substituted the last recorded blood pressure with the mean of the last 2 blood pressures on separate dates for the 2 health systems where those data were available (HS1 and HS3). Second, we excluded $\mathrm{BMI}$ from the primary model, as that was the most frequent missing variable, to ensure that BMI data were missing completely at random. Third, we developed models of risk factors for uncontrolled blood pressure restricted to those aged 18 to 40 and 40 to 60 years to examine whether similar risk factors were seen in both the young and middle aged. Finally we used $\geq 130 / 80 \mathrm{~mm} \mathrm{Hg}$ as the threshold for uncontrolled blood pressure at all ages, per ACC and AHA recommendations. ${ }^{9}$ Statistical testing was 2 sided, and significance was set at $P<.05$. All analyses were performed using SAS version 9.2 (SAS Institute, Cary, NC).

\section{Results}

\section{Characteristics of Patients with Hypertension}

Of 169,793 patients with at least 1 primary care visit in a 2 -year period in the 3 health networks, 
Table 1. Descriptive Characteristics of Patients with Hypertension in Three Health Systems

\begin{tabular}{|c|c|c|c|c|}
\hline Characteristic & Health System 1, n (\%) & Health System 2, n (\%) & Health System 3, n (\%) & Total, n (\%) \\
\hline $\mathrm{n}$ & 17,923 & 13,937 & 21,273 & 53,133 \\
\hline \multicolumn{5}{|l|}{ Age categories } \\
\hline 18 to 39 years & $851(5)$ & $1,072(8)$ & $2,024(10)$ & $3,847(7)$ \\
\hline 40 to 59 years & $7,264(41)$ & $4,926(35)$ & $10,514(49)$ & $22,704(43)$ \\
\hline 60 to 85 years & $9,808(55)$ & $7,939(57)$ & $8,743(41)$ & $26,482(50)$ \\
\hline Female sex & $8,880(50)$ & $7,317(53)$ & $12,076(57)$ & $28,273(53)$ \\
\hline \multicolumn{5}{|l|}{ Race/ethnicity } \\
\hline Non-Latino White & $3,214(18)$ & $5,848(42)$ & $6,197(29)$ & $15,250(30)$ \\
\hline African-American & $4,118(23)$ & $1,838(13)$ & $5,088(24)$ & $11,044(21)$ \\
\hline Latino & $3,113(17)$ & $1047(8)$ & $4,581(22)$ & $8,741(17)$ \\
\hline Asian/Pacific Islander & $5,976(33)$ & $3,807(27)$ & $4,420(21)$ & $14,203(27)$ \\
\hline American Indian or Alaska Native & $302(2)$ & $24(0)$ & $132(1)$ & $458(1)$ \\
\hline Other or unknown & $1,200(7)$ & $1,373(10)$ & $855(4)$ & $3,428(6)$ \\
\hline \multicolumn{5}{|l|}{ Preferred language } \\
\hline English & $10,560(59)$ & $11,661(84)$ & $15,376(72)$ & $3,7597(71)$ \\
\hline Spanish & $2,569(14)$ & $356(3)$ & 2,916 (14) & $5,841(11)$ \\
\hline Chinese & $2,832(16)$ & $823(6)$ & $264(1)$ & $3,919(7)$ \\
\hline Other $^{*}$ & $1,911(11)$ & $1,097(8)$ & $2,714(13)$ & $5,722(11)$ \\
\hline \multicolumn{5}{|l|}{ Insurance } \\
\hline Commercial & $1,241(7)$ & $6,815(49)$ & $6,117(29)$ & $14,173(27)$ \\
\hline Medicaid or local county coverage & $10,701(60)$ & $2,179(16)$ & $10,252(48)$ & $23,132(44)$ \\
\hline Medicare & $5,177(29)$ & $4,782(34)$ & $3,826(18)$ & $13,785(26)$ \\
\hline No health coverage & $804(4.5)$ & $161(1.2)$ & $1,078(5.1)$ & $2,043(8)$ \\
\hline \multicolumn{5}{|l|}{ Body mass index in $\mathrm{kg} / \mathrm{m}^{2}$} \\
\hline Less than 25 & $4,705(26)$ & $4,327(31)$ & $3,787(18)$ & $12,819(24)$ \\
\hline 25 to less than 30 & $5,832(33)$ & $4,794(34)$ & $6,181(29)$ & $16,807(32)$ \\
\hline 30 or greater & $6,803(38)$ & $4,638(33)$ & $10,475(49)$ & $21,916(41)$ \\
\hline Current smoker & $3,811(21)$ & $996(10)$ & $4,457(21)$ & $9,264(19)$ \\
\hline \multicolumn{5}{|l|}{ Comorbidities } \\
\hline Chronic kidney disease & $2,246(13)$ & $1,845(13)$ & $2,943(9)$ & $6,023(11)$ \\
\hline Diabetes mellitus & $6,207(35)$ & $4,111(30)$ & $7,803(37)$ & $18,121(34)$ \\
\hline Cardiovascular disease & $3,399(19)$ & 2,620 (19) & $2,350(11)$ & $8,369(16)$ \\
\hline \multicolumn{5}{|l|}{$\begin{array}{l}\text { Number of primary care visits } \\
\text { during 2-year period }\end{array}$} \\
\hline 1 to 2 visits & $2,493(14)$ & $1,385(10)$ & $2,250(11)$ & $6,128(12)$ \\
\hline 3 to 4 visits & $3,191(18)$ & $1,705(12)$ & 2,791(13) & $7,687(14)$ \\
\hline 5 or more & $12,153(68)$ & $10,847(78)$ & $16,232(76)$ & $39,232(74)$ \\
\hline $\begin{array}{l}\text { Blood pressure not controlled at last } \\
\text { visit }\end{array}$ & $5,110(29)$ & $4,337(31)$ & $9,304(44)$ & $18,751(35)$ \\
\hline
\end{tabular}

Information taken from electronic health records of all patients with $\geq 1$ primary care visit over 2 years.

$P$-values for all comparisons between systems were $<.01$ using $\chi^{2}$ tests.

*Most frequent other languages were Tagalog $(\mathrm{n}=1,070)$, Vietnamese $(\mathrm{n}=840)$, Russian $(\mathrm{n}=673)$, Punjabi $(\mathrm{n}=390)$, Farsi $(\mathrm{n}=$ 247), and Arabic $(\mathrm{n}=210)$.

$53,133(31 \%)$ met criteria for a diagnosis of hypertension. The proportion of patients with hypertension in each system was 34\% (HS1), 29\% (HS2), and $31 \%$ (HS3). Patients with hypertension were slightly more often female, and approximately half were aged 60 to 85 years. The profile of hypertensive patients varied between systems, with statisti- cally significant differences across all patient characteristics. Complete characteristic are displayed in Table 1.

\section{Risk Factors for Uncontrolled Blood Pressure}

Descriptive characteristics of patients with controlled and uncontrolled hypertension on the day 
of their last primary care visit are shown in Table 2. Overall, 18,751 (35.3\%) patients had uncontrolled blood pressure, with substantially higher proportions among those aged 40 to 59 years, of African-American race, reporting English as a preferred language, and from HS3. In a multivariate model (Table 3), several patterns emerged. Those aged 60 years or older without comorbidities had the lowest risk of uncontrolled blood pressure (target $<150 / 90 \mathrm{~mm}$ $\mathrm{Hg}$ ). African Americans had a higher risk of uncontrolled blood pressure, and Asian/Pacific Islanders had a lower risk of uncontrolled blood pressure compared with non-Latino whites; patients whose language preference was Cantonese or Mandarin also had a lower risk of uncontrolled blood pressure compared with those who preferred English. Having insurance of any type and more primary care visits were also independently associated with lower risk of uncontrolled blood pressure. Obesity and smoking were associated with higher risk of uncontrolled blood pressure. Risk of uncontrolled blood pressure varied independently by health system.

\section{Individual Clinic Comparisons}

Each health system included multiple primary care sites. There was significant variation in proportion of patients with uncontrolled blood pressure at their last visit, even after adjusting for the same patient factors as in Table 3. The probability of patients having uncontrolled blood pressure at each individual clinic, after adjusting for the same patient factors, is shown in Figure 1. Individual clinics ranged from having $16 \%$ (95\% CI, 14 to 17 ) to $48 \%$ (95\% CI, 46 to 50 ) of their hypertensive patients having uncontrolled blood pressure at their last visit. Even within the highest performing system, HS1, clinics ranged between 16\% (95\% CI, 14 to 17 ) and $39 \%$ (95\% CI, 36 to 41 ) of patients with uncontrolled blood pressure at their last primary care visit.

\section{Sensitivity Analyses}

In sensitivity analyses, taking an average of the last 2 blood pressures, which was available for 2 of the 3 health systems, did not result in substantially different results from using the most recent blood pressure measurement. Excluding BMI from the model did not result in substantially different outcomes in our multivariate models. When separate models were created for adults aged 18 to 40 and 40 to 60 years, similar patterns emerged without sig-
Table 2. Descriptive Characteristics of Patients with Controlled and Uncontrolled Blood Pressure on the Day of their Last Primary Care Visit $(n=53,133)$

\begin{tabular}{|c|c|c|}
\hline Characteristic & $\begin{array}{l}\text { Controlled, } \\
\text { n (\%) }\end{array}$ & $\begin{array}{c}\text { Uncontrolled, } \\
\mathrm{n}(\%)\end{array}$ \\
\hline $\mathrm{n}$ & 34,382 & 18,751 \\
\hline \multicolumn{3}{|l|}{ Age categories } \\
\hline 18 to 39 years & $2150(6)$ & $1797(10)$ \\
\hline 40 to 59 years & $13,520(39)$ & $9184(49)$ \\
\hline 60 to 85 years & $18,712(54)$ & $7770(41)$ \\
\hline Female sex & $18,529(54)$ & $9744(52)$ \\
\hline \multicolumn{3}{|l|}{ Race/ethnicity } \\
\hline Non-Latino white & $9805(29)$ & $5454(29)$ \\
\hline African-American & $6304(18)$ & $4740(25)$ \\
\hline Latino & $5595(16)$ & $3146(17)$ \\
\hline Asian/Pacific Islander & $10,404(30)$ & $4257(23)$ \\
\hline Native American & $316(1)$ & $142(1)$ \\
\hline Other or unknown & $1958(6)$ & $1,012(5)$ \\
\hline \multicolumn{3}{|l|}{ Language } \\
\hline English & $23,607(69)$ & $13,990(75)$ \\
\hline Spanish & $3842(11)$ & $1999(11)$ \\
\hline $\begin{array}{c}\text { Cantonese or } \\
\text { Mandarin }\end{array}$ & $3125(9)$ & $794(4)$ \\
\hline Others & $3775(11)$ & $1947(10)$ \\
\hline \multicolumn{3}{|l|}{ Insurance } \\
\hline $\begin{array}{l}\text { Insured or other } \\
\text { health coverage }\end{array}$ & $33,443(97)$ & $17,872(95)$ \\
\hline No health coverage & $1063(3)$ & $980(5)$ \\
\hline \multicolumn{3}{|l|}{ Body mass index $\left(\mathrm{kg} / \mathrm{m}^{2}\right)$} \\
\hline Less than 25 & $8949(27)$ & $5759(32)$ \\
\hline 25 to less than 30 & $14,411(43)$ & $7134(40)$ \\
\hline 30 or greater & $13,353(40)$ & $8563(48)$ \\
\hline Current smoker & $5289(17)$ & $3975(23)$ \\
\hline \multicolumn{3}{|l|}{$\begin{array}{l}\text { Number of primary care } \\
\text { visits during 2-year } \\
\text { period }\end{array}$} \\
\hline 1 to 2 visits & $3212(9)$ & $2916(16)$ \\
\hline 3 to 4 visits & $4761(14)$ & $2926(16)$ \\
\hline 5 or more visits & $26,361(77)$ & $12,871(69)$ \\
\hline \multicolumn{3}{|l|}{ Comorbidities } \\
\hline $\begin{array}{l}\text { Chronic kidney } \\
\text { disease }\end{array}$ & $3811(11)$ & $2212(12)$ \\
\hline Diabetes mellitus & $11,490(33)$ & $6631(35)$ \\
\hline Cardiovascular disease & $5516(16)$ & $2853(15)$ \\
\hline \multicolumn{3}{|l|}{ Health system } \\
\hline Health System 1 & $12,813(37)$ & $5110(27)$ \\
\hline Health System 2 & $9600(28)$ & $4337(23)$ \\
\hline Health System 3 & $11,969(35)$ & $9304(50)$ \\
\hline
\end{tabular}

Information taken from electronic health records of all patients with $\geq 1$ primary care visit over 2 years.

$P$-values for all comparisons between controlled and uncontrolled hypertension were $<.01$ using $\chi^{2}$ tests. 
Table 3. Multivariate Model of Risk of Uncontrolled Blood Pressure at the Most Recent Primary Care Visit

\begin{tabular}{|c|c|}
\hline Characteristic & Risk Ratio (95\% CI) \\
\hline \multicolumn{2}{|l|}{ Sex } \\
\hline Male (reference) & 1.0 \\
\hline Female & $0.98(0.96$ to 1.01$)$ \\
\hline \multicolumn{2}{|l|}{ Age/Comorbidity category* } \\
\hline $\begin{array}{l}\text { Aged }>60 \text { years with no } \\
\text { comorbidities (reference) }\end{array}$ & 1.0 \\
\hline Aged $\geq 60$ years with comorbidities & $1.64(1.57$ to 1.71$)$ \\
\hline $\begin{array}{l}\text { Aged }<60 \text { years with no } \\
\text { comorbidities }\end{array}$ & $1.72(1.65$ to 1.79$)$ \\
\hline Aged $<60$ years with comorbidities & $1.51(1.45$ to 1.58$)$ \\
\hline \multicolumn{2}{|l|}{ Race/ethnicity } \\
\hline Non-Latino white (reference) & 1.0 \\
\hline African American & $1.13(1.09$ to 1.16$)$ \\
\hline Latino & $1.01(0.96$ to 1.05$)$ \\
\hline Asian/Pacific Islander & $0.95(0.91$ to 0.98$)$ \\
\hline American Indian, other, or unknown & $0.99(0.93$ to 1.04$)$ \\
\hline \multicolumn{2}{|l|}{ Preferred language } \\
\hline English (reference) & 1.0 \\
\hline Spanish & $0.97(0.92$ to 1.02$)$ \\
\hline Cantonese or Mandarin & $0.79(0.73$ to 0.85$)$ \\
\hline Other language & $1.05(1.01$ to 1.10$)$ \\
\hline \multicolumn{2}{|l|}{ Insurance status } \\
\hline No health coverage (reference) & 1.0 \\
\hline Insured or other health coverage & $0.87(0.83$ to 0.92$)$ \\
\hline \multicolumn{2}{|l|}{ Smoking status } \\
\hline Never or past smoker (reference) & 1.0 \\
\hline Current smoker & $1.12(1.08$ to 1.15$)$ \\
\hline \multicolumn{2}{|l|}{ Body mass index (BMI) } \\
\hline $\begin{array}{l}\text { Not overweight or obese }(\mathrm{BMI}<25 \\
\left.\mathrm{kg} / \mathrm{m}^{2}\right) \text { (reference) }\end{array}$ & 1.0 \\
\hline Overweight (BMI 25 to $<30 \mathrm{~kg} / \mathrm{m}^{2}$ ) & $1.02(0.99$ to 1.06$)$ \\
\hline Obese $\left(\mathrm{BMI} \geq 30 \mathrm{~kg} / \mathrm{m}^{2}\right)$ & $1.09(1.06$ to 1.13$)$ \\
\hline \multicolumn{2}{|l|}{$\begin{array}{l}\text { Number of primary care visits over } \\
\text { 2-year period }\end{array}$} \\
\hline 1 to 2 visits (reference) & 1.0 \\
\hline 3 to 4 visits & $0.85(0.82$ to 0.88$)$ \\
\hline 5 or more visits & $0.71(0.69$ to 0.73$)$ \\
\hline \multicolumn{2}{|l|}{ Health System } \\
\hline Health System 1 (reference) & 1.0 \\
\hline Health System 2 & $1.15(1.11$ to 1.19$)$ \\
\hline Health System 3 & $1.46(1.42$ to 1.50$)$ \\
\hline
\end{tabular}

BMI, body mass index; CI, confidential interval.

Only patients with complete data were included $(n=51,417)$. Statistically significant risk ratio differences in comparison to reference variables are displayed in bold type.

*Comorbidities relevant to blood pressure target chronic kidney disease, diabetes, ans cardiovascular disease.

nificant differences between these 2 age groups. Finally, when defining uncontrolled blood pressure among hypertensive patients as $\geq 130 / 80 \mathrm{~mm} \mathrm{Hg}$, we found that the overall proportion of patients with uncontrolled blood pressure doubled from $35 \%$ to $70 \%$.

\section{Discussion}

We were able to construct a large database of primary care patients and their outcomes related to hypertension from 3 heterogeneous health systems participating in a data-sharing collaborative. Among over 50,000 diverse hypertensive patients, we found important disparities based not only on patient characteristics, but also between and within health systems.

This Hypertension Data Collaborative project demonstrates the challenges and opportunities of regional electronic data sharing across health systems using different EHRs systems. Only 3 of 7 health organizations invited were able to participate fully. Not surprisingly, the participating health care systems had more centralized decision-making processes and more robust internal informatics expertise. Nonetheless, the project facilitated the creation of shared research priorities, a common data dictionary, shared processes for EHR data extraction, transfer of the data into a shared data repository, data harmonization, and data analysis. Although created as a single data project without a solution for sustainable, longitudinal data sharing at this time, the project demonstrates the potential of such collaboration to inform future quality improvement and research interventions across heterogeneous health systems at the regional level.

Many of our findings related to blood pressure control among patients diagnosed with hypertension are consistent with the findings of others. ${ }^{13}$ Patients in the study cohort were more likely to have uncontrolled blood pressure if they were African Americans, current smokers, obese, had less frequent primary care visits, or no current health insurance coverage. What our findings add is that the same types of disparities are found consistently across different types of health settings with different demographic compositions.

We found that the patients aged 60 years and older without comorbidities were least likely to have uncontrolled blood pressure at their last visit. This finding likely reflects the higher target threshold for this group of patients $(<150 / 90 \mathrm{~mm} \mathrm{Hg})$, demonstrating how shifting blood pressure guidelines might influence which patients are targeted by 
Figure 1. Probability of hypertensive patients at each practice having uncontrolled blood pressure at their most recent primary care visit. Information taken from electronic health records of all patients with $\geq 1$ primary care visit over 2 years. Model adjusted for patient age, sex, race/ethnicity, insurance, obesity, smoking status, preferred language, number of primary care visits, and presence of comorbidities (cardiovascular disease, diabetes mellitus and chronic kidney disease). Error bars represent $95 \%$ CIs $(n=51,417)$. The vertical axis shows proportion of patients with hypertension, and the horizontal axis shows data for clinics within each health system. CI, confidential interval; Clin, Clinic.

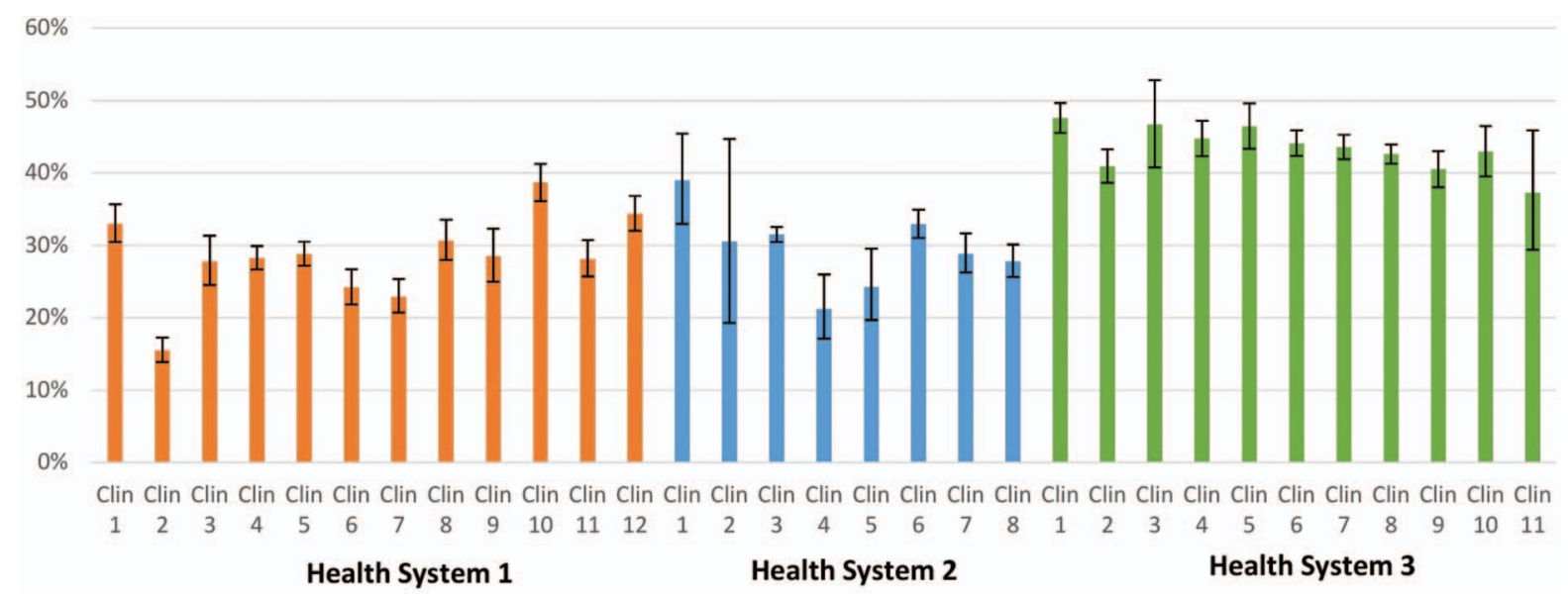

health system and/or whether health systems can achieve quality metrics. Notably, in our sensitivity analysis, we found that lowering the target for optimal blood pressure control to $<130 / 80 \mathrm{~mm} \mathrm{Hg}$ for all patients, per ACC/AHA guidelines, would double the number of hypertensive patients requiring additional attention to their blood pressure goals. This change would present a significant new challenge to health systems and demand additional resources for risk stratification and treatment of many hypertensive patients previously believed to have acceptable blood pressure control.

We also found significant differences in blood pressure control across these 3 health systems and between individual clinical sites within each health system. The health system with the lowest proportion of hypertensive patients with uncontrolled blood pressure (HS1) was a safety net system that has focused intensively on quality improvement initiatives to address hypertension control, at a time when neither of the other 2 health systems were implementing comprehensive hypertension quality improvement initiatives. The pilot site for this initiative, clinic 2 within HS1 (see Figure 1), started its work in 2014 and had a significantly lower proportion of hypertensive patients with uncontrolled blood pressure compared with the other clinics in this health system, which did not begin participation until the end of 2015. Results of this quality improvement initiative also indicate that this health system measurably increased the proportion of patients with controlled hypertension by the time the intervention was completed in 2016. These results suggest the potential benefits of hypertension quality improvement initiatives at the practice level and the opportunity for clinical teams both within and across health systems to benefit from sharing best practices. In addition, the highest performing health system was a safety net health system, suggesting that good hypertension control can be achieved in clinical settings with a significant proportion of low-income uninsured patients.

Our analyses are limited by several factors. First, there may be differences in the way that hypertension is measured, recorded, and diagnosed by individual clinicians within and across health systems, and this could be reflected in clinical data that is not collected following strictly validated and uniform protocols. In addition, comorbidities such as such as chronic kidney disease may not be accurately documented in EHRs, and with current guidelines used in this study, the systems with better documentation of comorbidities could seem to have worse blood pressure control. ${ }^{14}$ Other parameters such as current smoking status may not always be captured accurately in the EHRs, and other potentially important variables, such as current alcohol use, were not captured in this study. There may 
also be differences between health systems that agreed and declined participation in the data collaborative, making our sample less representative of hypertension control locally or elsewhere in the United States. These limitations are countered by the fact that our analyses are based on "real-world" clinical data that are available to clinical teams that manage hypertension at the point of care. The overall consistency of our findings across and within health systems supports the use of EHR data such as these to inform regional quality improvement and practice-based research interventions to address hypertension more effectively.

In conclusion, the Hypertension Data Collaborative successfully demonstrated the challenges and opportunities for electronic health data sharing and its use for identifying common disparities in hypertension care across 3 health systems. Future studies with this dataset will examine patterns of treatment with antihypertensive medications within these health systems to explore the relationship between medication underuse and deviation from guideline-based treatment algorithms. Sustainable approaches to support collection and aggregation of hypertension data longitudinally will be necessary to evaluate the impact of future interventions to address disparities that are identified.

The authors would like to acknowledge the clinical leaders and medical informatics teams at each health system without whose participation and assistance this research would not have been possible.

To see this article online, please go to: http://jabfm.org/content/ 31/6/897.full.

\section{References}

1. Registries for evaluating patient outcomes: A user's guide. In: Gliklich RE, Dreyer NA, Leavy MB, eds. Patient Registries. 1st ed. Rockville, MD: Agency for Healthcare Research and Quality (US); 2014 April.

2. Mozaffarian D, Benjamin EJ, Go AS. Heart disease and stroke statistics-2016 update: A report from the American Heart Association. Circulation. 2016;133: e38-e360.

3. Fryer CD, Ostchega Y, Hales CM, Zhang G, Kruszon-Moran D. Hypertension prevalence and control among adults: United States, 2015-2016. NCHS Data Brief No. 289, Oct 2017.
4. Egan BM, Li J, Small J, Nietert PJ, Sinopoli A. The growing gap in hypertension control between insured and uninsured adults: National Health and Nutrition Examination Survey 1988 to 2010. Hypertension 2014;64:997-1004.

5. Hyman DJ, Pavlik VN. Characteristics of patients with uncontrolled hypertension in the United States. N Engl J Med 2001;345:479-86.

6. Jaffe MG, Lee GA, Young JD, Sidney S, Go AS. Improved blood pressure control associated with a large-scale hypertension program. JAMA 2013;310: 699-705.

7. James PA, Oparil S, Carter BL, et al. 2014 evidencebased guideline for the management of high blood pressure in adults: Report from the panel members appointed to the Eighth Joint National Committee (JNC 8). JAMA 2014;311:507-20.

8. Qaseem A, Wilt TJ, Rich R, et al. Pharmacologic treatment of hypertension in adults aged 60 years or older to higher versus lower blood pressure targets: A clinical practice guideline from the American College Of Physicians and the American Academy of Family Physicians. Ann Intern Med 2017;166:430-7.

9. ACC/AHA/AAPA/ABC/ACPM/AGS/APhA/ASH/ ASPC/NMA/PCNA Guideline for the prevention, detection, evaluation, and management of high blood pressure in adults: A report of the American College of Cardiology/American Heart Association Task Force on Clinical Practice Guidelines. J Am Coll Cardiol 2018;12:579.e1-579.e73.

10. Cohen JB, Townsend RR. The ACC/AHA 2017 Hypertension guidelines: Both too much and not enough of a good thing? Ann Intern Med 2018;168: 287-8.

11. AAFP. AAFP decides not to endorse AHA/ACC hypertension guidelines. Available from: https://www. aafp.org/news/health-of-the-public/20171212 notendorseaha-accgdlne.html.

12. Knol MJ, Le Cessie S, Algra A, Vandenbroucke JP, Greonwold RH. Overestimation of risk rations by odds ratios in trials and cohort studies: Alternatives to logistic regression. CMAJ 2012;184:895-9.

13. Gu Q, Burt VL, Dillon CF, Yoon S. Trends in antihypertensive medication use and blood pressure control among United States adults with hypertension: The National Health and Nutrition Examination Survey, 2001 to 2010. Circulation 2012;126: 2105-14.

14. Cipparone CW, Withiam-Leitch M, Kimminau KS, Fox CH, Singh R, Kahn L. Inaccuracy of ICD-9 Codes for chronic kidney disease: A study from two practice-based Research Networks (PBRNs). J Am Board Fam Med 2015;28:678-82. 